\title{
Alteration of gene expression and DNA methylation in drug-resistant gastric cancer
}

\author{
OSAMU MAEDA ${ }^{1}$, TAKAFUMI ANDO ${ }^{2}$, NAOKI OHMIYA ${ }^{3}$, KAZUHIRO ISHIGURO $^{1}$, OSAMU WATANABE $^{2}$, \\ RYOJI MIYAHARA ${ }^{2}$, YOKO HIBI ${ }^{4}$, TAKU NAGAI ${ }^{4}$, KIYOFUMI YAMADA $^{4}$ and HIDEMI GOTO ${ }^{2}$ \\ Departments of ${ }^{1}$ Advanced Research of Gastroenterology and ${ }^{2}$ Gastroenterology and Hepatology, Nagoya University \\ Graduate School of Medicine, Showa-ku, Nagoya; ${ }^{3}$ Department of Gastroenterology, School of Medicine, Fujita Health \\ University, Toyoake, Aichi; ${ }^{4}$ Department of Hospital Pharmacy, Nagoya University Hospital, Nagoya, Japan
}

Received December 10, 2013; Accepted January 15, 2014

DOI: $10.3892 /$ or.2014.3014

\begin{abstract}
The mechanisms of drug resistance in cancer are not fully elucidated. To study the drug resistance of gastric cancer, we analyzed gene expression and DNA methylation profiles of 5-fluorouracil (5-FU)- and cisplatin (CDDP)-resistant gastric cancer cells and biopsy specimens. Drug-resistant gastric cancer cells were established with culture for $>10$ months in a medium containing 5-FU or CDDP. Endoscopic biopsy specimens were obtained from gastric cancer patients who underwent chemotherapy with oral fluoropyrimidine S-1 and CDDP. Gene expression and DNA methylation analyses were performed using microarray, and validated using real-time PCR and pyrosequencing, respectively. Out of 17,933 genes, 541 genes commonly increased and 569 genes decreased in both 5-FU- and CDDP-resistant AGS cells. Genes with expression changed by drugs were related to GO term 'extracellular region' and 'p53 signaling pathway' in both 5-FU- and CDDP-treated cells. Expression of 15 genes including KLK13 increased and 12 genes including ETV7 decreased, in both drug-resistant cells and biopsy specimens of two patients after chemotherapy. Out of 10,365 genes evaluated with both expression microarray and methylation microarray, 74 genes were hypermethylated and downregulated, or hypomethylated and upregulated in either 5-FU-resistant or CDDP-resistant cells. Of these genes, expression of 21 genes including FSCN1, CPT1C and NOTCH3, increased from treatment with a demethylating agent. There are alterations of gene expression and DNA methylation in drug-resistant gastric cancer; they may be related to mechanisms of drug resistance and may be useful as biomarkers of gastric cancer drug sensitivity.
\end{abstract}

Correspondence to: Dr Osamu Maeda, Department of Advanced Research of Gastroenterology, Nagoya University Graduate School of Medicine, 65 Tsurumai-cho, Showa-ku, Nagoya 466-8550, Japan E-mail: maeda-o@med.nagoya-u.ac.jp

Key words: gastric cancer, cisplatin, 5-fluorouracil, drug resistance, DNA methylation

\section{Introduction}

Gastric cancer is one of the most common causes of cancer-related mortality, responsible for $>700,000$ deaths worldwide per year (1). Although the main treatment strategy for gastric cancer is surgical or endoscopic resection, unresectable cases are treated with systemic chemotherapy. Platinum agents and fluoropyrimidine are the key therapeutic drugs for advanced gastric cancer (2) and drug resistance is an important problem accompanying treatment. A number of studies have previously reported on the mechanisms of gastric cancer chemoresistance (3) using cultured cells, animal models and clinical tissue samples. However, the mechanisms of drug resistance have not been fully elucidated.

It has been reported that both genetic and epigenetic changes play important roles in carcinogenesis and tumor progression (4). In various types of cancer, epigenetic changes are known to be early events in the multi-steps of carcinogenesis (5). Promoter hypermethylation is well-known to be important for the suppression of tumor suppressor gene expression (4). Mechanisms of cancer drug resistance are considered to be multifactorial; they have epigenetic alterations (6) and involve multiple gene functions and signaling pathways. A better understanding of such mechanisms may provide therapeutic strategies for gastric cancer.

In the present study, drug-resistant gastric cancer cell lines were established, and biopsy specimens were obtained from patients after the acquisition of drug resistance. Genome-wide analysis of gene expression and DNA methylation with a microarray for drug-resistant cell lines and endoscopic biopsy specimens of gastric cancer was performed. Validation with quantitative methods was also performed.

\section{Materials and methods}

Cell culture and 5-aza-2'-deoxycytidine (5-aza-dC) treatment. AGS was purchased from the American Type Culture Collection (ATCC) (Manassas, VA, USA), and cultured in RPMI-1640 medium with $10 \% \mathrm{FBS}$ at $37^{\circ} \mathrm{C}$ with $5 \% \mathrm{CO}_{2}$. For treatment with 5-aza-dC (decitabine), cells were seeded on day 0 , and exposed to freshly prepared $10 \mu \mathrm{mol} / 15$-aza-dC (Sigma-Aldrich, Tokyo, Japan) for $24 \mathrm{~h}$ on days 1 and 3. After 
each treatment, the cells were placed in fresh medium and harvested on day 4 (7).

Drug-resistant gastric cancer cells. Resistant AGS cells were generated by continuous exposure to increasing concentrations of cisplatin (CDDP) or 5-fluorouracil (5-FU) for 10 months. Viability of cells was measured by MTS-formazan reduction using CellTiter 96 Aqueous One Solution Cell Proliferation Assay (Promega, Madison, WI, USA). AGS cells $\left(2 \times 10^{3}\right)$ were cultured using 96-well microplates for $24 \mathrm{~h}$, and exposed to various concentrations of CDDP or $5-\mathrm{FU}$ for $48 \mathrm{~h}$ to calculate the $\mathrm{IC}_{50}$ of CDDP or 5-FU for each cell line.

Patients and biopsy specimens. Endoscopic biopsy specimens were obtained from two patients with unresectable advanced gastric cancer who underwent 3 and 4 courses of chemotherapy with oral fluoropyrimidine S-1 plus CDDP (8) before and after treatment. Samples after chemotherapy were obtained from lesions with viable cancer. The present study was approved by the Ethics Committee of Nagoya University Graduate School of Medicine, and written informed consent was provided by the patients.

Extraction of DNA and RNA from cell lines and gastric biopsy specimens. Cells or biopsy specimens were stored at $-80^{\circ} \mathrm{C}$ for DNA extraction, and we used RNAlater (Ambion, Austin, TX, USA) for RNA extraction. For extraction of DNA and RNA, DNA Mini kit (Qiagen, Venlo, The Netherlands) and RNA Mini kit (Qiagen) were used, respectively.

Gene expression analysis with microarray. Expression analysis was performed with SurePrint G3 Human GE 8x60K (Agilent, Loveland, CO, USA). Expression of mRNA of autosomal 17,933 genes was evaluated with 23,856 corresponding probes. A difference in signal intensity $>2$-fold was judged to be significant.

DNA methylation microarray. Bisulfite-converted DNA was used for hybridization on Infinium HumanMethylation450 BeadChip (Illumina, San Diego, CA, USA). The $\beta$-value [intensity of the methylated allele (M)/(intensity of the unmethylated allele (U) + intensity of the methylated allele $(\mathrm{M})+100)$ ] was calculated for each $\mathrm{CpG}$ site (9). Methylation levels of candidate promoter lesions in $\mathrm{CpG}$ islands of 11,692 genes were evaluated. Genes with a difference in their $\beta$-value $>0.1$ were extracted. Of these 11,692 genes, expression of 10,365 genes was also evaluated with expression microarray.

Gene ontology (GO) and pathway analysis. GO analysis was performed with TargetMine (http://targetmine.nibio. go.jp/targetmine/begin.do; National Institute of Biomedical Innovation, Osaka, Japan), and pathway analysis was performed with the Kyoto Encyclopedia of Genes and Genomes (KEGG) database. GO terms or pathways with $\mathrm{P}<0.05$ using the HolmBonferroni method were judged to be significantly enriched.

Real-time PCR. Real-time PCR was performed to validate expression of mRNA with TaqMan Gene Expression Assays and TaqMan Gene Expression Master Mix (both from Applied Biosystems, Foster City, CA, USA).
Bisulfite pyrosequencing. Bisulfite treatment was performed with the EpiTect kit (Qiagen) according to the manufacturer's protocol. PyroMark PCR (Qiagen) was used to perform PCR, and bisulfite pyrosequencing was performed as previously reported (10-12). In brief, the biotinylated PCR product was captured on streptavidin-coated beads (Amersham Biosciences, Uppsala, Sweden) and run on the PSQHS Pyrosequencing System (Biotage, Uppsala, Sweden) to obtain the degree of methylation.

\section{Results}

Resistance to CDDP or 5-FU of established drug-resistant cell lines. To confirm whether gastric cancer cells cultured in chemotherapy agents obtained drug resistance, $\mathrm{IC}_{50}$ values were measured. $\mathrm{IC}_{50}$ values of 5-FU were 10 and $56 \mu \mathrm{M}$ in parent AGS and 5-FU-resistant AGS (5FUr), respectively. $\mathrm{IC}_{50}$ values of CDDP were 13 and $25 \mu \mathrm{M}$ in parent AGS and CDDP-resistant AGS (CDDPr), respectively.

Genes with altered expression in drug-resistant cells and biopsy specimens. To characterize gene expression profiles of drug-resistant gastric cancer cells, expression microarray analysis was performed. A comparison of parent AGS, 5FUr and CDDPr change of expression is shown in Fig. 1. The expression of 541 genes increased and the expression of 569 genes decreased in both 5FUr and CDDPr compared with parent AGS. In contrast, the expression of only 25 genes increased in 5FUr and decreased in CDDPr, and the expression of only seven genes decreased in 5FUr and increased in CDDPr. To examine the characterization of genes with expression altered by drug treatment, we performed GO analysis and pathway analysis (Table I). Although most enriched GO terms differed between those changed in 5FUr and those changed in CDDPr, 'extracellular region' in GO terms of cellular component was commonly enriched in both 5FUr and CDDPr. With pathway analysis, the 'p53 signaling pathway' was enriched in both 5FUr and CDDPr.

To compare gene expression of gastric cancer before and after chemotherapy, expression microarray analysis with endoscopic biopsy specimens was performed. Genes with altered expression both in drug-resistant cells and in biopsy specimens after chemotherapy were extracted. The expression of 15 genes increased 5FUr, CDDPr and two pairs of biopsy specimens, and the expression of 12 genes decreased (Fig. 2, Table II).

To validate the gene expression change extracted with microarray, real-time PCR for KLK13 and ETV7 was performed. Consistent with microarray analysis, KLK13 increased in both drug-resistant AGS cells and endoscopic biopsy specimens after chemotherapy (Fig. 3A). In contrast, ETV7 decreased in both drug-resistant cells and biopsy specimens after treatment (Fig. 3B).

Integrated analysis of expression and methylation microarray. To study whether DNA methylation contributes to gene expression change caused by chemotherapy agents, we analyzed the methylation profiles of 5FUr and CDDPr and compared them with parent AGS. The number of genes that were hypermethylated and decreased in expression and that were hypomethylated and increased in expression was 74 . 

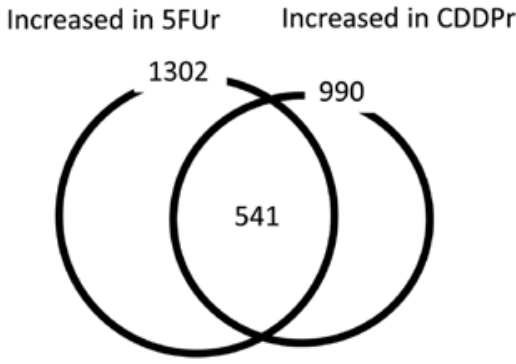

Increased in 5FUr Decreased in CDDPr

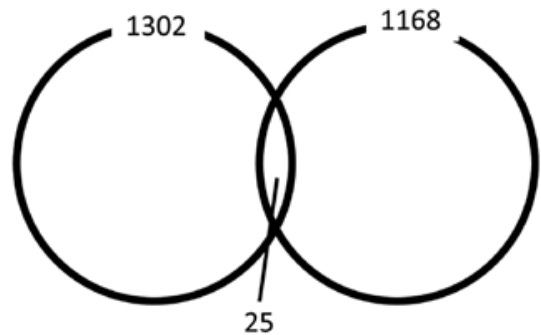

Decreased in 5FUr Decreased in CDDPr

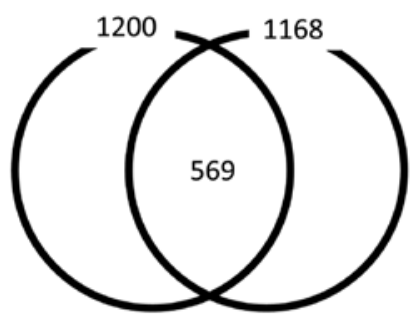

Decreased in 5FUr Increased in CDDPr

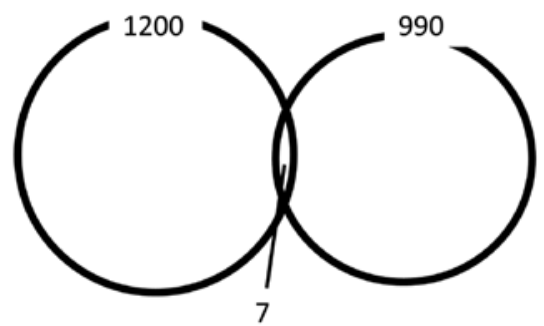

Figure 1. Venn diagrams showing number of genes in which expression increased or decreased with 5-FU or CDDP treatment. 5FUr, 5-fluorouracil-resistant AGS cells; CDDPr, cisplatin-resistant AGS cells.

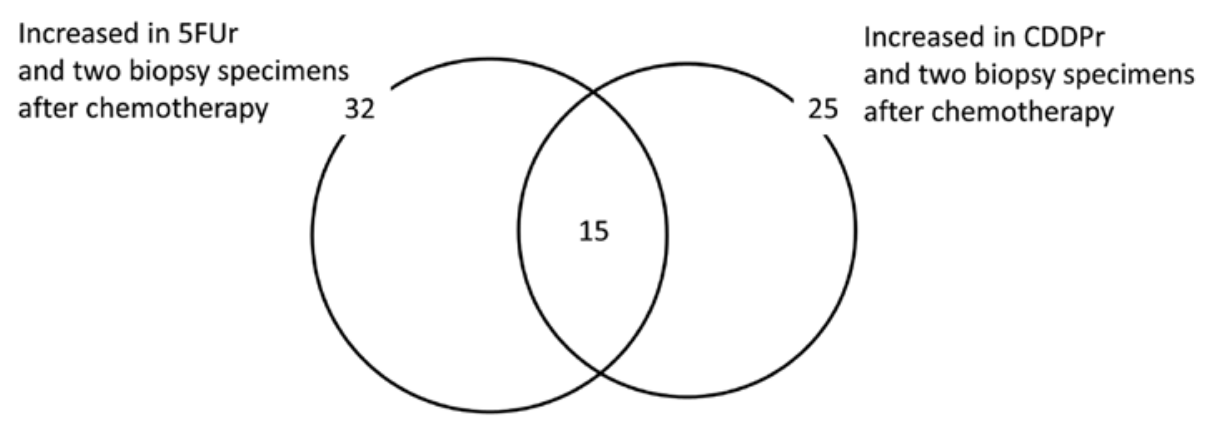

Decreased in 5FUr and two biopsy specimens after chemotherapy

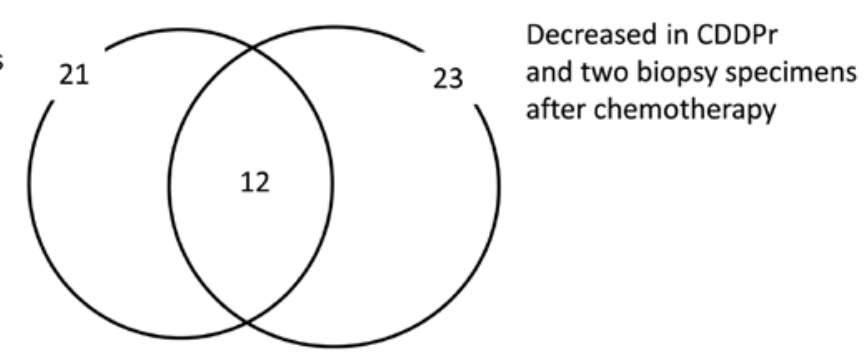

Figure 2. Venn diagrams showing number of genes in which expression was commonly changed in drug-resistant cells and biopsy specimens. 5FUr, 5-fluorouracil-resistant AGS cells; CDDPr, cisplatin-resistant AGS cells.

Next, to evaluate whether alterations in the DNA methylation of these genes was related to expression change, gene expression change was measured by treatment with a demethylating agent. Twenty-one of those 74 genes increased in expression after treatment with decitabine (Table III). Furthermore, gene expression and methylation were validated with quantitative methods, TaqMan PCR and bisulfite pyrosequencing, respectively, for FSCN1, CPT1C and NOTCH3.
Expression of these three genes increased after treatment with decitabine (Fig. 4). FSCN1 revealed increased expression and hypomethylation in CDDPr compared with parent AGS cells. Regarding CPT1C, 5FUr showed hypomethylation and increased expression. CDDPr also showed increased expression, although the methylation level did not change. NOTCH3 showed increased expression and hypomethylation, especially in $5 \mathrm{FUr}$. 
Table I. Significantly enriched gene ontology (GO) terms and pathways of genes in which expression was changed in 5-fluorouracil-resistant AGS cells (5FUr) and cisplatin-resistant AGS cells (CDDPr), compared with parent AGS cells.

A, GO terms (biological processes)

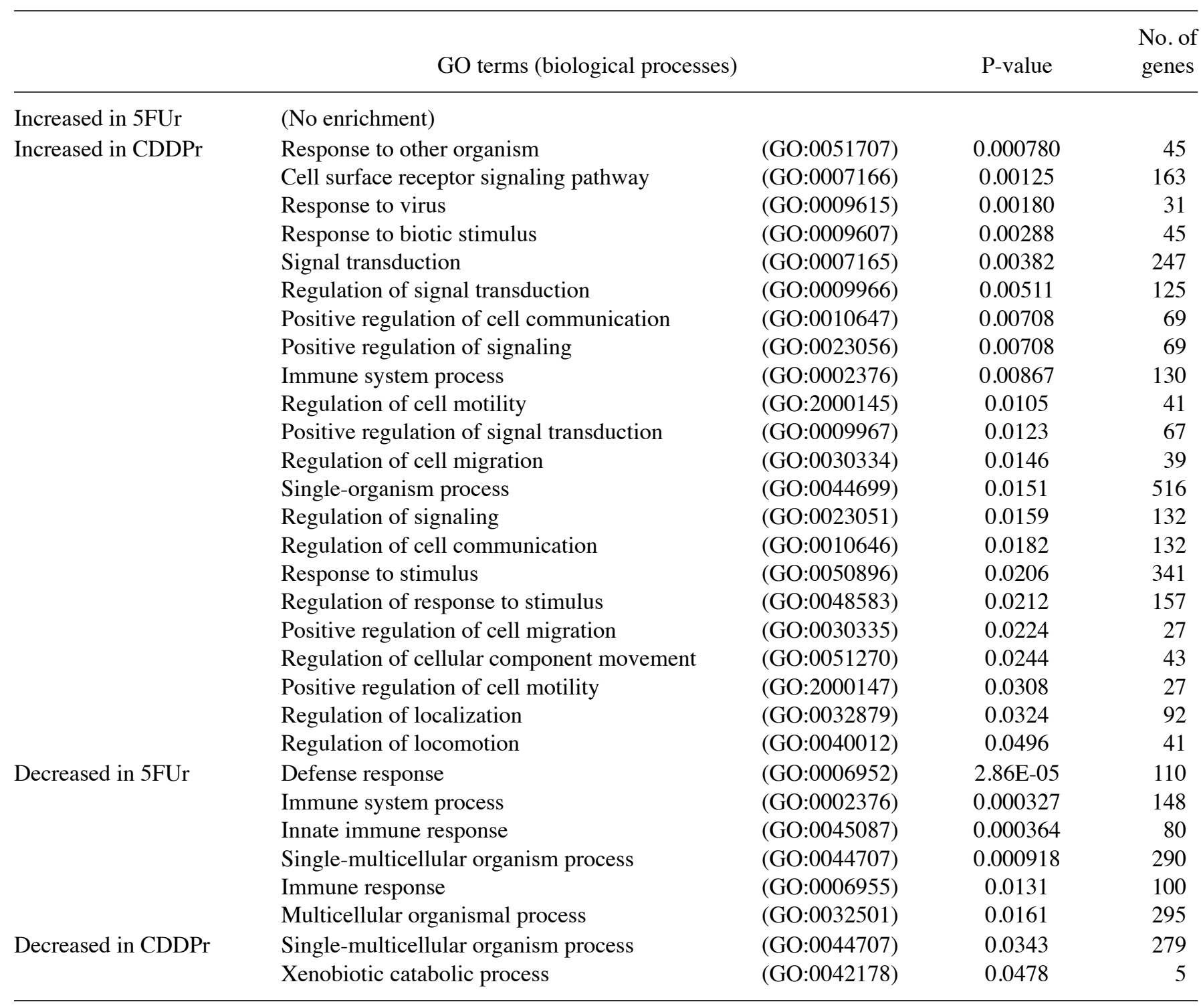

B, GO terms (cellular components)

GO terms (cellular components)

No. of $\mathrm{P}$-value genes

\begin{tabular}{ll}
\hline Increased in 5FUr & Extracellular region \\
& Cell periphery \\
& Plasma membrane \\
& Extracellular region part \\
& Plasma membrane part \\
& Extracellular space \\
& Extracellular region \\
Increased in CDDPr & Extracellular region \\
Decreased in 5FUr & Extracellular region \\
Decreased in CDDPr & Cornified envelope \\
& Intrinsic to membrane \\
& Integral to membrane \\
& Cell periphery \\
& Plasma membrane
\end{tabular}

(GO:0005576)

(GO:0071944)

(GO:0005886)

(GO:0044421)

(GO:0044459)

(GO:0005615)

(GO:0005576)

(GO:0005576)

(GO:0005576)

(GO:0001533)

(GO:0031224)

(GO:0016021)

(GO:0071944)

(GO:0005886)

$\begin{array}{cr}4.64 \mathrm{E}-07 & 130 \\ 0.000754 & 280 \\ 0.000907 & 277 \\ 0.00647 & 70 \\ 0.0134 & 136 \\ 0.0271 & 56 \\ 0.0374 & 92 \\ 3.24 \mathrm{E}-05 & 116 \\ 7.37 \mathrm{E}-08 & 121 \\ 0.0124 & 8 \\ 0.0175 & 142 \\ 0.0195 & 138 \\ 0.0202 & 243 \\ 0.0268 & 240\end{array}$


Table I. Continued.

$\mathrm{C}, \mathrm{GO}$ terms (molecular functions)

\begin{tabular}{|c|c|c|c|c|}
\hline & GO terms (molecular fun & & P-value & $\begin{array}{l}\text { No. of } \\
\text { genes }\end{array}$ \\
\hline \multirow[t]{3}{*}{ Increased in $5 \mathrm{FUr}$} & Serine-type peptidase activity & (GO:0008236) & $3.29 \mathrm{E}-05$ & 24 \\
\hline & Serine hydrolase activity & (GO:0017171) & $5.16 \mathrm{E}-05$ & 24 \\
\hline & Serine-type endopeptidase activity & (GO:0004252) & 0.00325 & 17 \\
\hline Increased in CDDPr & (No enrichment) & & & \\
\hline Decreased in $5 F U r$ & (No enrichment) & & & \\
\hline \multirow[t]{2}{*}{ Decreased in CDDPr } & Receptor binding & (GO:0005102) & $5.08 \mathrm{E}-04$ & 83 \\
\hline & Cytokine activity & (GO:0005125) & 0.0383 & 15 \\
\hline \multicolumn{5}{|l|}{ D, pathway } \\
\hline \multicolumn{3}{|c|}{ Pathway } & P-value & $\begin{array}{l}\text { No. of } \\
\text { genes }\end{array}$ \\
\hline Increased in $5 \mathrm{FUr}$ & \multicolumn{2}{|l|}{ p53 signaling pathway } & 0.0222 & 14 \\
\hline Increased in CDDPr & \multicolumn{2}{|l|}{ p53 signaling pathway } & $8.56 \mathrm{E}-07$ & 18 \\
\hline Decreased in 5FUr & \multicolumn{2}{|l|}{ (No enrichment) } & & \\
\hline Decreased in CDDPr & \multicolumn{2}{|l|}{ Cytokine-cytokine receptor interaction } & 0.00618 & 37 \\
\hline
\end{tabular}
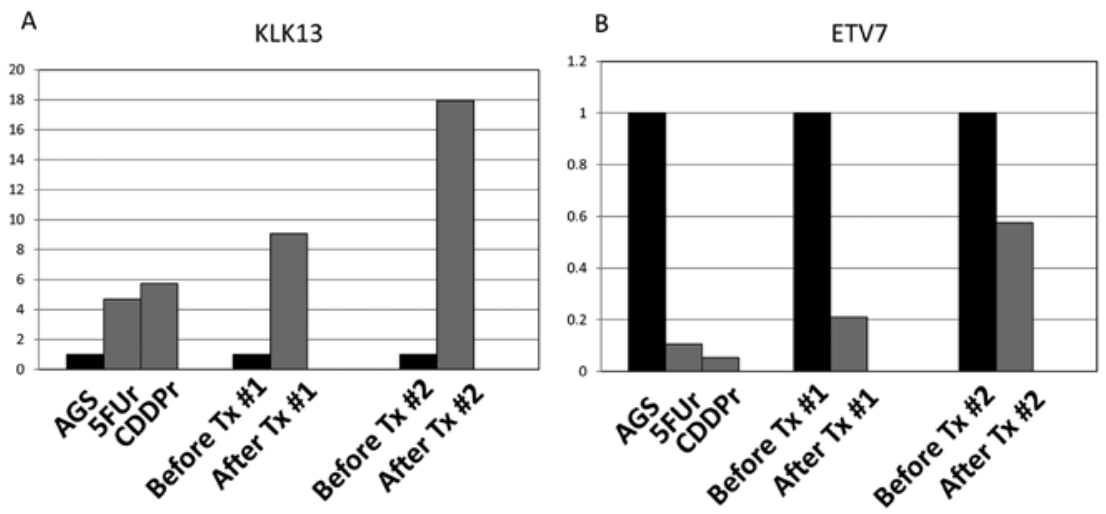

Figure 3. Relative expression levels in (A) KLK13 and (B) ETV7 in drug-resistant cells and biopsy specimens. Relative levels of parent AGS and biopsy specimens before treatment were set to one. Tx, chemotherapy; 5FUr, 5 -fluorouracil-resistant AGS cells; CDDPr, cisplatin-resistant AGS cells.

\section{Discussion}

The gene expression and the DNA methylation of drug-resistant cell lines and biopsy specimens were evaluated before and after chemotherapy, and some genes revealed altered expression and altered methylation. In drug-resistant cells, treatment with 5-FU and CDDP caused consistent expression change in $>1,000$ genes. In contrast, the expression of only a small number of genes changed reciprocally (Fig. 1), and those genes were considered to be potentially related with drugspecific sensitivity. In GO analysis, only a small number of GO terms are commonly enriched in both 5-FU-resistant cells and CDDP-resistant cells, and enriched pathways related to the two drugs are also different. These findings may be related to differences in mechanisms of resistance to each drug.
Expression change of genes before and after chemotherapy was also evaluated using endoscopic biopsy specimens, and revealed that profiles of changes were different in the two patients. Expression of some genes increased or decreased, both in drug-resistant cells and biopsy specimens after chemotherapy (Fig. 2, Table II). Such genes are considered to be candidates as key molecules for drug resistance, and may be useful as biomarkers of drug-sensitivity.

KLK13 is one member of the tissue kallikrein (KLK) family which includes 15 genes (KLK1-KLK15) and plays a role in tumor cell invasion and migration (13). KLK13 has already been reported to be upregulated in gastric cancer cells after exposure to antineoplastic agents, including epirubicin and methotrexate (14). It has also been reported that overexpression of KLK13 results in an increase of malignant 
Table II. List of genes in which expression was changed commonly in drug-resistant cells and biopsy specimens.

\begin{tabular}{|c|c|c|c|c|c|}
\hline $\begin{array}{l}\text { Increased in } 5 \mathrm{FUr} \\
\text { and two biopsy } \\
\text { specimens after } \\
\text { chemotherapy }\end{array}$ & $\begin{array}{l}\text { Decreased in 5FUr } \\
\text { and two biopsy } \\
\text { specimens after } \\
\text { chemotherapy }\end{array}$ & $\begin{array}{l}\text { Increased in CDDPr } \\
\text { and two biopsy } \\
\text { specimens after } \\
\text { chemotherapy }\end{array}$ & $\begin{array}{l}\text { Decreased in CDDPr } \\
\text { and two biopsy } \\
\text { specimens after } \\
\text { chemotherapy }\end{array}$ & $\begin{array}{l}\text { Increased in } \\
\text { 5FUr, CDDPr, } \\
\text { and two biopsy } \\
\text { specimens after } \\
\text { chemotherapy }\end{array}$ & $\begin{array}{l}\text { Decreased in } \\
5 F U r, \text { CDDPr, } \\
\text { and two biopsy } \\
\text { specimens after } \\
\text { chemotherapy }\end{array}$ \\
\hline APOC1 & ALPK 1 & ACTG2 & ACOXL & APOC1 & ALPK1 \\
\hline BAIAP3 & C17orf110 & ANPEP & ALPK1 & CRYM & CCL21 \\
\hline C4BPA & C4orf47 & APOC1 & BATF & DNAJC28 & CYP2E1 \\
\hline C6orf154 & CCL21 & C9orf123 & BEST4 & HSD17B6 & ETV7 \\
\hline CAPS2 & CYP2E1 & CELA3B & CCL21 & IQCD & FBXO15 \\
\hline CFTR & ETV7 & CRYM & CYP2E1 & KLK13 & GPR110 \\
\hline CRYM & FBXO15 & CTSG & ETV7 & KREMEN2 & NLRC5 \\
\hline DNAJC28 & GPR110 & DNAJC28 & FBXO15 & OLFML3 & PLIN4 \\
\hline FRZB & HEPACAM2 & HOXB3 & GPR110 & OTUD7A & SLC22A20 \\
\hline HSD17B6 & IFI44L & HSD17B6 & INSC & PHACTR3 & SLC26A9 \\
\hline IGF1 & KRT6C & IQCD & KRT31 & PLAT & SLC28A3 \\
\hline IP6K3 & LAMC2 & KLK13 & MUC1 & RARRES2 & SNORA22 \\
\hline IQCD & NLRC5 & KREMEN2 & NCKAP5 & SRI & \\
\hline KCTD7 & OR52K2 & NLRP2 & NLRC5 & TAC3 & \\
\hline KLK13 & PLIN4 & NRG1 & PCSK9 & TNNI3 & \\
\hline KREMEN2 & SLC22A20 & OLFML3 & PLIN4 & & \\
\hline LAMA1 & SLC26A9 & OTUD7A & RAB27B & & \\
\hline LRRC6 & SLC28A3 & PHACTR3 & SLC22A20 & & \\
\hline MSLN & SNORA22 & PLAT & SLC26A9 & & \\
\hline NR2F1 & SPRR3 & RARRES2 & SLC28A3 & & \\
\hline OLFML3 & ZNF750 & RASL10A & SLFNL1 & & \\
\hline OOEP & & SRI & SNORA22 & & \\
\hline OTUD7A & & TAC3 & SYT13 & & \\
\hline PHACTR3 & & TNFSF9 & & & \\
\hline PLAT & & TNNI3 & & & \\
\hline \multicolumn{6}{|l|}{ PRODH } \\
\hline \multicolumn{6}{|l|}{ RARRES2 } \\
\hline \multicolumn{6}{|l|}{ SCN2A } \\
\hline \multicolumn{6}{|l|}{ SEPP1 } \\
\hline \multicolumn{6}{|l|}{ SRI } \\
\hline \multicolumn{6}{|l|}{ TAC3 } \\
\hline TNNI3 & & & & & \\
\hline
\end{tabular}

5FUr, 5-fluorouracil-resistant AGS cells; CDDPr, cisplatin-resistant AGS cells.

cell behavior, and that knockdown of its endogenous gene expression causes a significant decrease in cell migratory and invasive properties (13). We found that KLK13 increased in both drug-resistant cells and biopsy specimens, a finding suggesting that KLK13 may play a role in 5-FU and CDDP resistance in gastric cancer.

In contrast to KLK13, expression of ETV7 decreased in drug-resistant cells. ETV7 is a member of the Ets transcription factor family and is reported to act as an inhibitor of differentiation (15). Since ETV7 was downregulated in drug-resistant gastric cancer in the present study, it may be related with mechanisms of gastric cancer drug-sensitivity.
It has been reported that epigenetic profiles are useful for identifying molecular mediators for cancer drug sensitivity (6). In terms of a correlation between gene expression and DNA methylation, we also performed expression and methylation microarray analyses, and found some genes with altered methylation levels and expression levels. FSCN1 revealed increased expression and hypomethylation in CDDPresistant cells. FSCN1 has been reported to play an important role in cancer development and is associated with invasion and metastasis (16). It has also been reported that higher intensity FSCN1 staining correlated with more-advanced cancer stages, and inversely correlated with survival rates in 
Table III. List of genes in which expression increased and methylation levels decreased, or expression decreased and methylation levels increased.

\begin{tabular}{|c|c|c|c|}
\hline $\begin{array}{l}\text { Increased } \\
\text { expression } \\
\text { and hypo- } \\
\text { methylation } \\
\text { in 5FUr }\end{array}$ & $\begin{array}{l}\text { Decreased } \\
\text { expression } \\
\text { and hyper- } \\
\text { methylation } \\
\text { in 5FUr }\end{array}$ & $\begin{array}{l}\text { Increased } \\
\text { expression } \\
\text { and hypo- } \\
\text { methylation } \\
\text { in CDDPr }\end{array}$ & $\begin{array}{l}\text { Decreased } \\
\text { expression } \\
\text { and hyper- } \\
\text { methylation } \\
\text { in CDDPr }\end{array}$ \\
\hline ARC & ATP2C2 & ABCG4 & ATP2C2 \\
\hline C12orf34 & C15orf60 & C12orf34 & C15orf60 \\
\hline CPT1C & PRAME & CARD9 & FRMD6 \\
\hline CST6 & ZNF773 & CST6 & SECTM1 \\
\hline CYB5R2 & & FES & TNFSF12 \\
\hline KCNH8 & & FSCN1 & ZNF773 \\
\hline MESP1 & & KCNH8 & \\
\hline NOTCH3 & & MESP1 & \\
\hline RASGRP2 & & VGF & \\
\hline TNFSF12 & & & \\
\hline
\end{tabular}

5FUr, 5-fluorouracil-resistant AGS cells; CDDPr, cisplatin-resistant AGS cells.

gastric adenocarcinoma (17). This suggests that FSCN1 may influence patient survival through acquisition of resistance to chemotherapy drugs.

In our experiment, CPT1C was increased in expression in 5-FU and CDDP-resistant cells, and demethylated in 5-FU-resistant cells. CPT1C has been reported to promote tumor growth and rapamycin resistance (18). CPT1C expression correlates inversely with mammalian target of rapamycin (mTOR) pathway activation, contributes to rapamycin resistance in murine primary tumors, and is frequently upregulated in human lung tumors (18). To our knowledge, there has been no report of a relationship between CPT1C and 5-FU or CDDP.

Notch is a transmembrane heterodimeric receptor with 4 distinct members (NOTCH1 to NOTCH4) present in humans. NOTCH3 is one of the Notch family members. It has been reported that NOTCH1, another molecule in Notch family members, expression is associated with cell aggressiveness and 5-FU drug resistance in human esophageal squamous cell carcinoma cell lines in vitro, and also with poor survival in human esophageal squamous cell carcinomas (19). It has also been reported that expression levels of Notch3 were increased in rat tracheal epithelial cells after treatment with 5-FU (20). NOTCH3 knockdown enhanced the sensitivity of nasopharyngeal carcinoma cells to CDDP treatment (21), and NOTCH3 overexpression correlated with shorter progressionfree/overall survival in patients with advanced stage ovarian carcinoma treated with platinum and taxane (22). In our data, NOTCH3 was upregulated in drug-resistant cells. In gastric cancer, NOTCH3 may be related to drug resistance.

However, we could not find a significant difference in methylation levels among biopsy samples. One of the limitations is that biopsy specimens may not represent the characteristics of the whole tumor, since gastric cancer is known to be biologically heterogeneous.
$\mathbf{A}$

FSCN1
Expression
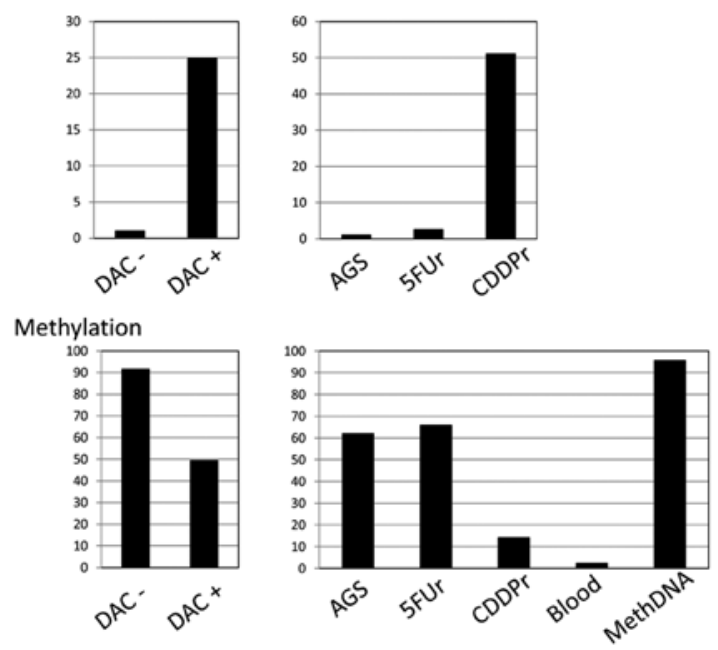

B

CPT1C

Expression
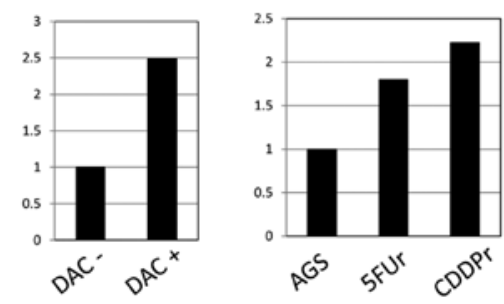

Methylation
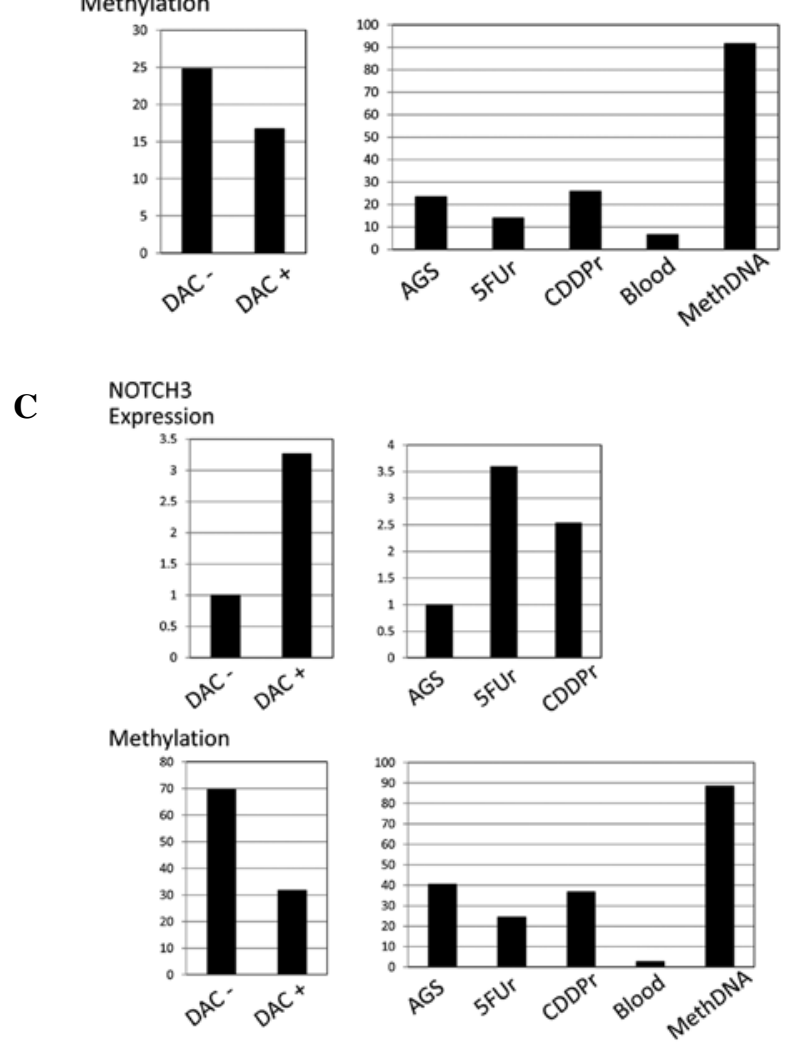

Figure 4. Expression and methylation levels of (A) FSCN1, (B) CPT1C and (C) NOTCH3. Note that AGS cells without treatment with a chemotherapy agent are passaged at the same time as the established drug-resistant cells. Methylation levels may differ between untreated AGS cells using the decitabine treatment experiment and the experiment of comparison with drug-resistant cells. DAC, demethylating agent 5-aza-dC; MethDNA, methylated DNA for positive control; 5FUr, 5-fluorouracil-resistant AGS cells; CDDPr, cisplatin-resistant AGS cells. 
In the present study, genes with altered expression and DNA methylation were extracted after treatment with chemotherapeutic agents in gastric cancer. These alterations may be related to mechanisms of gastric cancer drug resistance, and may be useful as biomarkers that predict drug sensitivity. Further studies with a large number of clinical samples are necessary.

\section{Acknowledgements}

The authors thank Ms. Chie Moriyama for her technical support. This study was funded by the Ministry of Education, Culture, Sports, Science and Technology of Japan (no. 20378053).

\section{References}

1. Ferlay J, Shin HR, Bray F, Forman D, Mathers C and Parkin DM: Estimates of worldwide burden of cancer in 2008: GLOBOCAN 2008. Int J Cancer 127: 2893-2917, 2010.

2. Okines A, Verheij M, Allum W, Cunningham D, Cervantes A; ESMO Guidelines Working Group: Gastric cancer: ESMO Clinical Practice Guidelines for diagnosis, treatment and follow-up. Ann Oncol 21 (Suppl 5): v50-v54, 2010.

3. Zhang D and Fan D: New insights into the mechanisms of gastric cancer multidrug resistance and future perspectives. Future Oncol 6: 527-537, 2010.

4. Taby R and Issa JP: Cancer epigenetics. CA Cancer J Clin 60: 376-392, 2010.

5. Ushijima T: Epigenetic field for cancerization. J Biochem Mol Biol 40: 142-150, 2007.

6. Shen L, Kondo Y, Ahmed S, et al: Drug sensitivity prediction by $\mathrm{CpG}$ island methylation profile in the NCI- 60 cancer cell line panel. Cancer Res 67: 11335-11343, 2007.

7. Yamashita S, Takahashi S, McDonell N, et al: Methylation silencing of transforming growth factor- $\beta$ receptor type II in rat prostate cancers. Cancer Res 68: 2112-2121, 2008.

8. Koizumi W, Narahara H, Hara T, et al: S-1 plus cisplatin versus S-1 alone for first-line treatment of advanced gastric cancer (SPIRITS trial): a phase III trial. Lancet Oncol 9: 215-221, 2008

9. Bibikova M, Barnes B, Tsan C, et al: High density DNA methylation array with single $\mathrm{CpG}$ site resolution. Genomics 98: 288-295, 2011.
10. Colella S, Shen L, Baggerly KA, Issa JP and Krahe R: Sensitive and quantitative universal Pyrosequencing methylation analysis of CpG sites. Biotechniques 35: 146-150, 2003.

11. An B, Kondo Y, Okamoto Y, et al: Characteristic methylation profile in $\mathrm{CpG}$ island methylator phenotype-negative distal colorectal cancers. Int J Cancer 127: 2095-2105, 2010.

12. Shen L, Guo Y, Chen X, Ahmed S and Issa JP: Optimizing annealing temperature overcomes bias in bisulfite PCR methylation analysis. Biotechniques 42: 48-58, 2007.

13. Chou RH, Lin SC, Wen HC, Wu CW and Chang WS: Epigenetic activation of human kallikrein 13 enhances malignancy of lung adenocarcinoma by promoting $N$-cadherin expression and laminin degradation. Biochem Biophys Res Commun 409: 442-447, 2011.

14. Florou D, Mavridis K and Scorilas A: The kallikrein-related peptidase $13(K L K 13)$ gene is substantially up-regulated after exposure of gastric cancer cells to antineoplastic agents. Tumour Biol 33: 2069-2078, 2012

15. Gu X, Shin BH, Akbarali Y, et al: Tel-2 is a novel transcriptional repressor related to the Ets factor Tel/ETV-6. J Biol Chem 276: 9421-9436, 2001.

16. Hanker LC, Karn T, Holtrich U, et al: Prognostic impact of fascin-1 (FSCN1) in epithelial ovarian cancer. Anticancer Res 33: 371-377, 2013.

17. Tsai WC, Jin JS, Chang WK, et al: Association of cortactin and fascin-1 expression in gastric adenocarcinoma: correlation with clinicopathological parameters. J Histochem Cytochem 55: 955-962, 2007.

18. Reilly PT and Mak TW: Molecular pathways: tumor cells co-opt the brain-specific metabolism gene $C P T 1 C$ to promote survival. Clin Cancer Res 18: 5850-5855, 2012.

19. Liu J, Fan H, Ma Y, et al: Notch1 is a 5-fluorouracil resistant and poor survival marker in human esophagus squamous cell carcinomas. PLoS One 8: e56141, 2013.

20. Ma XB, Jia XS, Liu YL, et al: Expression and role of Notch signalling in the regeneration of rat tracheal epithelium. Cell Prolif 42: 15-28, 2009.

21. Man CH, Wei-Man Lun S, Wai-Ying Hui J, et al: Inhibition of NOTCH3 signalling significantly enhances sensitivity to cisplatin in EBV-associated nasopharyngeal carcinoma. J Pathol 226: 471-481, 2012.

22. Rahman MT, Nakayama K, Rahman M, et al: Notch3 overexpression as potential therapeutic target in advanced stage chemoresistant ovarian cancer. Am J Clin Pathol 138: 535-544, 2012. 